\title{
Species diversity of opisthobranch molluscs on Lizard Island, Great Barrier Reef, Australia
}

\author{
Heike Wägele ${ }^{1}$, Ingo Burghardt ${ }^{2}$, Nils Anthes ${ }^{3}$, Jussi Evertsen ${ }^{4}$, \\ Annette Klussmann-Kolb ${ }^{5}$ and Gilianne Brodie ${ }^{6}$ \\ 'Museum König Bonn und Institut für Evolutionsbiologie, An der Immenburg 1, 53121 Bonn, Germany, \\ hwaegele@evolution.uni-bonn de \\ Spezielle Zoologie, Ruhr-Universität Bochum, 44780 Bochum, Germany \\ Animal Evolutionary Ecology, Zoological Institute, University of Tübingen, \\ Auf der Morgenstelle 28, 72076 Tübingen, Germany \\ ' Trondhjem Biological Station, Norwegian University of Science and Technology, 7491 Trondheim, Norway \\ Zoologisches Institut, J.W. Goethe Universität, Siesmayerstrasse70, 60054 Frankfurt, Germany \\ - Institute of Applied Sciences, University of the South Pacific, P.O. Box 1168, Suva, Fiji.
}

\begin{abstract}
During several visits from 1999 to 2004 to the Australian Museum Research Station on Lizard Island (LIRS) (Great Barrier Reef, Northern Section, Queensland, Australia) the authors collected 158 different opisthobranch species from the reefs and lagoon areas surrounding Lizard Island. The number of species found gives an estimate of the diversity of the opisthobranch fauna on the northern Great Barrier Reef. The manuscript also provides colour plates of most species found so far on Lizard Island that will facilitate species identification, data usage and further data collection.
\end{abstract}

Key words: Biodiversity, Opisthobranchia, Mollusca, Australia, Lizard Island, Great Barrier Reef

\section{INTRODUCTION}

Coral reefs have more species per unit area than any other marine ecosystem but, with the exception of a few groups such as fishes and corals, most taxa are poorly known (Done et al. 1996). However, the wealth of coral reef biodiversity lies in small, cryptic invertebrates and there is a need for systematic inventories that address these types of fauna (Mikkelsen and Cracraft 2001). This was convincingly shown by an investigation of a coral reef in New Caledonia, where several discrete stations were intensively collected and a total of 2738 molluscan species were found, including 258 opisthobranchs (Bouchet et al. 2002).

Although faunal investigations of the Great Barrier Reef (GBR) are very important, for a better understanding of marine biodiversity and conservation on Australian coral reefs, many taxa are still inadequately understood with respect to their species diversity, seasonal and geographic distribution. Despite their often-dazzling appearance, this holds true for the Opisthobranchia, a relatively well known and diverse group of marine gastropod molluscs. The annotated work of Marshall and Willan (1999) on the opisthobranchs of Heron Island (at the southern end of the GBR Marine Park) is an unusual exception and is of great assistance for many scientists working to identify and document information related to tropical opisthobranch species. In addition, several popular books with good photographs of opisthobranchs (seaslugs) are available and give an accurate impression of the general morphology and diversity of these fascinating animals. These publications usually cover both the Indonesian and Australian regions (e.g. Willan and Coleman 1984, Coleman 2001).

The Great Barrier Reef is the largest coral reef ecosystem in the world and has been designated both a Marine Park and a World Heritage Area (Chadwick and Green 2002). Our aim is to contribute to the knowledge of biodiversity and distribution of the opisthobranch mollusc fauna on the Great Barrier Reef by summarizing our data from several scientific visits to Lizard Island Research Station over a 5-year period. Lizard Island is a continental island surrounded by fringing reefs and is situated very close to the northern section of the GBR Marine Park, approximately $1200 \mathrm{~km}$ north of Heron Island where the work of Marshall and Willan (1999) was conducted. This is the first time that a complete inventory of collected opisthobranchs from Lizard Island is presented. 
Table 1 Species list of Lizard Island's opisthobranchs. HW and GB during July 1999 and July 2002, AKK in July 1999, IB during July to September 2002 and August to September 2004, NA during January to February 2003 and October 2003 to February 2004, and JE during July 2003. The species collected in the aquaria of the research station were probably sucked in by the seawater system, which is supplied via an inlet pipe in front of the research station (Casuarina Beach) in a depth of about 1 to $2 \mathrm{~m}$. The last column indicates further published information on the material. Some species are described with a general Indo Pacific distribution but only localities north of the GBR. When no literature is known to us with the GBR explicitly mentioned as locality, we consider the findings on Lizard Island as new to the GBR, despite the general assumption of broad distribution. This is indicated with a $+\mathrm{R}+$ indicates, that this species is mentioned in Bill Rudman's sea slug forum at the Australian Museum in Sydney (www.seaslugforum.net) to occur in the GBR. For the new species, a question mark indicates that distribution is not known, but this species is probably new to the GBR

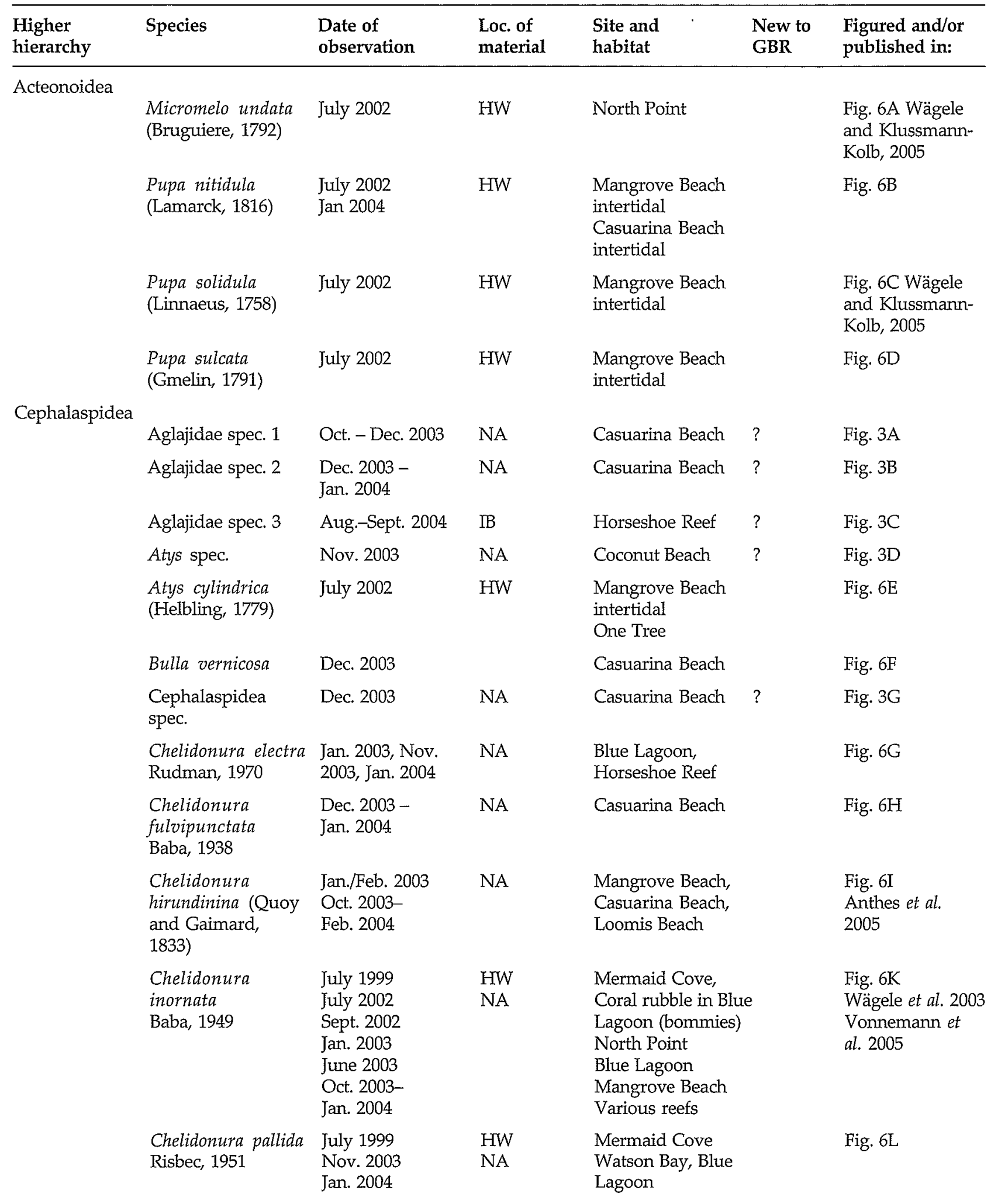


Table 1 (cont.)

\begin{tabular}{|c|c|c|c|c|c|c|}
\hline $\begin{array}{l}\text { Higher } \\
\text { Hierarchy }\end{array}$ & Species & $\begin{array}{l}\text { Date of } \\
\text { Observation }\end{array}$ & $\begin{array}{l}\text { Loc. of } \\
\text { material }\end{array}$ & $\begin{array}{l}\text { Site and } \\
\text { Habitat }\end{array}$ & $\begin{array}{l}\text { New to } \\
\text { GBR }\end{array}$ & $\begin{array}{l}\text { Figured and/or } \\
\text { published in: }\end{array}$ \\
\hline & $\begin{array}{l}\text { Chelidonura } \\
\text { sandrana } \\
\text { Rudman, } 1973\end{array}$ & $\begin{array}{l}\text { Jan. } 2003 \\
\text { Oct. } 2003 \\
\text { - Feb. } 2004 \\
\text { Aug.-Sept. } 2004\end{array}$ & $\begin{array}{l}\text { NA } \\
\text { IB }\end{array}$ & $\begin{array}{l}\text { Blue lagoon, } \\
\text { Casuarina Beach } \\
\text { Loomis Reef, } \\
\text { Casuarina Beach }\end{array}$ & + & $\begin{array}{l}\text { Fig. } 6 \mathrm{M}, \mathrm{N} \\
\text { Anthes and } \\
\text { Michiels } 2005 \\
\text { Anthes et al. } 2006\end{array}$ \\
\hline & $\begin{array}{l}\text { Chelidonura varians } \\
\text { Eliot, } 1903\end{array}$ & $\begin{array}{l}\text { July } 1999 \\
\text { June } 2003 \\
\text { Nov. } 2003 \\
\text { - Jan. } 2004\end{array}$ & $\begin{array}{l}\text { HW } \\
\text { NA }\end{array}$ & $\begin{array}{l}\text { Coral rubble in } \\
\text { Blue Lagoon } \\
\text { Ghost Beach, } \\
\text { Cobia Hole } \\
\text { Blue lagoon }\end{array}$ & $\mathrm{R}+$ & Fig. 60 \\
\hline & $\begin{array}{l}\text { Gastropteron } \\
\text { bicornutum Baba } \\
\text { and Tokioka, } 1965\end{array}$ & Jan. 2004 & NA & $\begin{array}{l}\text { Casuarina Beach } \\
\text { coral sand }\end{array}$ & + & Fig. 7A \\
\hline & Gastropteron spec. & July 2002 & $\mathrm{HW}$ & $\begin{array}{l}\text { Bird Island inside } \\
\text { the Blue Lagoon }\end{array}$ & $?$ & Fig. 3E \\
\hline & $\begin{array}{l}\text { Haminoea } \\
\text { cymbalum } \\
\text { (Quoy and } \\
\text { Gaimard, 1833) }\end{array}$ & $\begin{array}{l}\text { Jan. } 2003 \\
\text { Dec. } 2003\end{array}$ & & \multicolumn{2}{|l|}{ Off Casuarina Beach } & Fig. 7B \\
\hline & $\begin{array}{l}\text { Melanochlamys } \\
\text { spec. }\end{array}$ & $\begin{array}{l}\text { Jan. } 2003, \\
\text { Oct. } 2003 \\
\text { - Jan. } 2004\end{array}$ & NA & $\begin{array}{l}\text { Horseshoe Reef, } \\
\text { coral sand } \\
\text { Blue Lagoon, } \\
\text { Casuarina Beach }\end{array}$ & $?$ & $\begin{array}{l}\text { Fig. 3F } \\
\text { Melanochlamys } \\
\text { spec.1 in } \\
\text { Marshall and } \\
\text { Willan } 1999\end{array}$ \\
\hline & $\begin{array}{l}\text { Phanerophthalmus } \\
\text { smaragdinus } \\
\text { (Rüppell and } \\
\text { Leuckart, 1830) }\end{array}$ & $\begin{array}{l}\text { July } 1999 \\
\text { Aug.-Sept. } 2004\end{array}$ & $\begin{array}{l}\text { HW } \\
\text { IB }\end{array}$ & \multicolumn{2}{|l|}{$\begin{array}{l}\text { Ghost Beach } \\
\text { intertidal } \\
\text { Coconut Beach }\end{array}$} & $\begin{array}{l}\text { Fig. } 7 \mathrm{C} \\
\text { Vonnemann } \\
\text { et al. } 2005 \\
\text { Wägele and } \\
\text { Klussmann-Kolb, } \\
2005\end{array}$ \\
\hline & $\begin{array}{l}\text { Philinopsis cyanea } \\
\text { (Martens, 1879) }\end{array}$ & $\begin{array}{l}\text { Jan./Feb. } 2003 \\
\text { Oct. } 2003 \\
\text { - Jan. } 2004\end{array}$ & $\mathrm{NA}$ & \multicolumn{2}{|l|}{$\begin{array}{l}\text { Off Casuarina Beach, } \\
\text { Loomis Reef, } \\
\text { Mangrove Beach } \\
\text { Blue Lagoon, } \\
\text { Casuarina Beach }\end{array}$} & Fig. 7D \\
\hline & $\begin{array}{l}\text { Philinopsis } \\
\text { gardineri } \\
\text { (Eliot, 1903) }\end{array}$ & $\begin{array}{l}\text { Jan./Febr. } 2003 \\
\text { June } 2003 \\
\text { Nov. } 2003 \\
\text { - Jan. } 2004\end{array}$ & NA & \multicolumn{2}{|l|}{$\begin{array}{l}\text { Coconut Beach, } \\
\text { South Island } \\
\text { Off Casuarina } \\
\text { Beach, Palfrey Island } \\
\text { Blue Lagoon }\end{array}$} & $\begin{array}{l}\text { Fig. 7E } \\
\text { Wägele and } \\
\text { Klussmann-Kolb, } \\
2005\end{array}$ \\
\hline & $\begin{array}{l}\text { Philinopsis } \\
\text { lineolata (H. and } \\
\text { A. Adams, 1854) }\end{array}$ & $\begin{array}{l}\text { Dec. } 2003 \\
\text { - Jan. } 2004\end{array}$ & NA & \multicolumn{2}{|l|}{$\begin{array}{l}\text { Casuarina Beach, } \\
\text { Osprey Islet } \\
\text { coral rubble }\end{array}$} & Fig. TH \\
\hline & $\begin{array}{l}\text { Philinopsis } \\
\text { pilsbryi } \\
\text { (Eliot, 1900) }\end{array}$ & Jan. 2004 & NA & \multicolumn{2}{|l|}{$\begin{array}{l}\text { Bird Islet, } \\
\text { coral rubble }\end{array}$} & Fig. 7G \\
\hline & $\begin{array}{l}\text { Philinopsis } \\
\text { reticulata } \\
\text { (Eliot, 1903) }\end{array}$ & $\begin{array}{l}\text { Jan. } 2003 \\
\text { June } 2003 \\
\text { Nov. } 2003 \\
\text { - Jan. } 2004 \\
\text { Aug.-Sept. } 2004\end{array}$ & $\begin{array}{l}\text { NA } \\
\text { IB }\end{array}$ & $\begin{array}{l}\text { Off Casuarina } \\
\text { Beach, Loomis Reef, } \\
\text { Mangrove Beach, } \\
\text { Watson Bay South } \\
\text { Island, Bird Island, } \\
\text { Palfrey Island, } \\
\text { Casuarina Beach }\end{array}$ & $\mathrm{R}+$ & Fig. 7F \\
\hline & $\begin{array}{l}\text { Sagaminopteron } \\
\text { ornatum Tokioka } \\
\text { and Baba, } 1964\end{array}$ & $\begin{array}{l}\text { July } 1999, \\
\text { July } 2002 \\
\text { Jan. } 2004\end{array}$ & $\begin{array}{l}\text { HW } \\
\text { NA }\end{array}$ & \multicolumn{2}{|l|}{$\begin{array}{l}\text { Off Bird Island } \\
\text { outer reef, Coral } \\
\text { rubble in Blue } \\
\text { Lagoon (bommies), } \\
\text { Coral head Blue } \\
\text { Lagoon }\end{array}$} & $\begin{array}{l}\text { Fig. 7I } \\
\text { Wägele and } \\
\text { Klussmann-Kolb, } \\
2005\end{array}$ \\
\hline
\end{tabular}




\begin{tabular}{|c|c|c|c|c|c|c|}
\hline $\begin{array}{l}\text { Higher } \\
\text { Hierarchy }\end{array}$ & Species & $\begin{array}{l}\text { Date of } \\
\text { Observation }\end{array}$ & $\begin{array}{l}\text { Loc. of } \\
\text { material }\end{array}$ & $\begin{array}{l}\text { Site and } \\
\text { Habitat }\end{array}$ & $\begin{array}{l}\text { New to } \\
\text { GBR }\end{array}$ & $\begin{array}{l}\text { Figured and/or } \\
\text { published in: }\end{array}$ \\
\hline & $\begin{array}{l}\text { Siphopteron } \mathrm{cf} . \\
\text { pohnpei }\end{array}$ & $\begin{array}{l}\text { Oct. } 2003 \\
\text { - Jan. } 2004\end{array}$ & NA & $\begin{array}{l}\text { Casuarina Beach, } \\
\text { Watson Bay }\end{array}$ & & Fig. 3H \\
\hline & $\begin{array}{l}\text { Siphopteron } \\
\text { quadrispinosum } \\
\text { Gosliner, } 1989\end{array}$ & $\begin{array}{l}\text { Oct. } 2003 \\
\text { - Jan. } 2004\end{array}$ & $\mathrm{NA}$ & Casuarina Beach & + & Fig. 7K \\
\hline & $\begin{array}{l}\text { Siphopteron cf. } \\
\text { tigrinum } \\
\text { Gosliner, } 1989\end{array}$ & Sept. 2002 & $\mathrm{HW}$ & North Point & & Fig. 7L \\
\hline \multirow[t]{6}{*}{ Anaspidea } & $\begin{array}{l}\text { Aplysia } \\
\text { dactylomela } \\
\text { Rang, } 1828\end{array}$ & July 2002 & HW & $\begin{array}{l}\text { Coconut Beach } \\
\text { intertidal }\end{array}$ & & \\
\hline & $\begin{array}{l}\text { Aplysia paroula } \\
\text { Guilding in } \\
\text { Morch, } 1863 .\end{array}$ & Jan. 2004 & & Horseshoe Reef & & Fig. 8A \\
\hline & $\begin{array}{l}\text { Bursatella } \\
\text { leachii de } \\
\text { Blainville, } 1817\end{array}$ & Dec. 2003 & & North Point & & Fig. 8B \\
\hline & $\begin{array}{l}\text { Dolabella } \\
\text { auricularia } \\
\text { (Lightfoot, 1786) }\end{array}$ & $\begin{array}{l}\text { July } 2002 \\
\text { June } 2003 \\
\text { Dec. } 2003\end{array}$ & $\mathrm{HW}$ & $\begin{array}{l}\text { Coconut Beach } \\
\text { intertidal } \\
\text { Coconut Beach } \\
\text { Lizard Aquarium }\end{array}$ & & Fig. 8C \\
\hline & $\begin{array}{l}\text { Notarchus } \\
\text { indicus } \\
\text { Schweigger, } 1820\end{array}$ & Feb 2003 & & LIRS, Aquarium & & Fig. 8D \\
\hline & $\begin{array}{l}\text { Stylocheilus } \\
\text { striatus (Quoy } \\
\text { and Gaimard, } \\
\text { 1832) }\end{array}$ & $\begin{array}{l}\text { Sept. } 2002 \\
\text { Jan. } 2003 \\
\text { June } 2003 \\
\text { Dec. } 2003\end{array}$ & & $\begin{array}{l}\text { LIRS, Aquarium } \\
\text { Off Casuarina } \\
\text { Beach }\end{array}$ & & $\begin{array}{l}\text { Fig. 8E } \\
\text { We follow the } \\
\text { discussion in } \\
\text { Rudman } \\
\text { (seaslugforum) } \\
\text { and consider the } \\
\text { species found } \\
\text { here as S. striatus }\end{array}$ \\
\hline \multirow[t]{6}{*}{ Sacoglossa } & $\begin{array}{l}\text { Costasiella } \\
\text { usagi Ichikawa, } \\
1993\end{array}$ & $\begin{array}{l}\text { Dec. } 2003 \\
\text { - Jan. } 2004\end{array}$ & NA & Watson Bay & + & Fig. 8F \\
\hline & $\begin{array}{l}\text { Costasiella cf } \\
\text { ocelligera }\end{array}$ & $\begin{array}{l}\text { Dec. } 2003 \\
\text { - Jan. } 2004\end{array}$ & NA & $\begin{array}{l}\text { Watson Bay, } \\
\text { Casuarina Beach }\end{array}$ & $?$ & Fig. 4F \\
\hline & $\begin{array}{l}\text { Cyerce nigricans } \\
\text { (Pease, 1866) }\end{array}$ & $\begin{array}{l}\text { July } 1999 \\
\text { Aug. } 2002 \\
\text { Jan. } 2004\end{array}$ & $\mathrm{HW}$ & $\begin{array}{l}\text { Coconut Beach } \\
\text { intertidal } \\
\text { Casuarina Beach } \\
\text { coral sand }\end{array}$ & & $\begin{array}{l}\text { Fig. } 8 G \\
\text { Wägele and } \\
\text { Johnson } 2001 \\
\text { Vonnemann } \\
\text { et al } 2005\end{array}$ \\
\hline & Cyerce spec. 1 & July 2002 & HW & $\begin{array}{l}\text { Coconut Beach } \\
\text { intertidal }\end{array}$ & $?$ & Fig. 4A \\
\hline & Cyerce spec. 2 & $\begin{array}{l}\text { Sept. } 2002 \\
\text { Aug.-Sept. } 2004\end{array}$ & $\begin{array}{l}\text { HW } \\
\text { IB }\end{array}$ & $\begin{array}{l}\text { Between Bird } \\
\text { and South Island } \\
\text { intertidal }\end{array}$ & $?$ & Fig. 4B \\
\hline & $\begin{array}{l}\text { Elysia cf. } \\
\text { amakusana Baba, } \\
1955\end{array}$ & $\begin{array}{l}\text { Dec. } 2003 \\
\text { Jan. } 2004\end{array}$ & NA & $\begin{array}{l}\text { Blue Lagoon } \\
\text { Mangrove Beach }\end{array}$ & + & Fig. $8 \mathrm{H}$ \\
\hline
\end{tabular}


Table 1 (cont.)

\begin{tabular}{|c|c|c|c|c|c|c|}
\hline $\begin{array}{l}\text { Higher } \\
\text { Hierarchy }\end{array}$ & Species & $\begin{array}{l}\text { Date of } \\
\text { Observation }\end{array}$ & $\begin{array}{l}\text { Loc. of } \\
\text { material }\end{array}$ & $\begin{array}{l}\text { Site and } \\
\text { Habitat }\end{array}$ & $\begin{array}{l}\text { New to } \\
\text { GBR }\end{array}$ & $\begin{array}{l}\text { Figured and/or } \\
\text { published in: }\end{array}$ \\
\hline & $\begin{array}{l}\text { Elysia bennettae } \\
\text { Thompson, } 1973\end{array}$ & $\begin{array}{l}\text { July } 1999 \\
\text { July } 2002\end{array}$ & $\mathrm{HW}$ & $\begin{array}{l}\text { Ghost Beach } \\
\text { intertidal }\end{array}$ & & $\begin{array}{l}\text { Fig. } 81 \\
\text { Wägele and } \\
\text { Johnson } 2001 \text { as } \\
\text { E. expansa }\end{array}$ \\
\hline
\end{tabular}

\begin{tabular}{|c|c|}
\hline $\begin{array}{l}\text { Elysia ornata } \\
\text { (Swainson, 1840) }\end{array}$ & $\begin{array}{l}\text { Apr. } 1990 \\
\text { July } 1999 \\
\text { July } 2002 \\
\text { Aug. } 2002 \\
\text { June } 2003 \\
\text { Jan. 2004 } \\
\text { Aug.-Sept. } 2004\end{array}$ \\
\hline Elysia spec. & $\begin{array}{l}\text { Aug. } 2002 \\
\text { Aug.-Sept. } 2004\end{array}$ \\
\hline $\begin{array}{l}\text { Elysia tomentosa } \\
\text { Jensen, } 1997\end{array}$ & $\begin{array}{l}\text { Sept. } 2002 \\
\text { Aug.-Sept. } 2004\end{array}$ \\
\hline $\begin{array}{l}\text { Elysiella pusilla } \\
\text { Bergh, } 1872\end{array}$ & $\begin{array}{l}\text { July } 1999 \\
\text { July } 2002 \\
\text { June } 2003\end{array}$ \\
\hline $\begin{array}{l}\text { Oxynoe viridis } \\
\text { (Pease, 1861) }\end{array}$ & $\begin{array}{l}\text { June } 2003 \\
\text { Jan. } 2004\end{array}$ \\
\hline $\begin{array}{l}\text { Plakobranchus } \\
\text { ocellatus } \\
\text { Hasselt, } 1824\end{array}$ & $\begin{array}{l}\text { July } 1999 \\
\text { July } 2002 \\
\text { June } 2003 \\
\text { Dec. } 2003 \\
\text { - Jan. } 2004 \\
\text { Aug.-Sept. } 2004\end{array}$ \\
\hline
\end{tabular}

$\begin{array}{lll}\text { HW } & \text { Blue Lagoon, } & \text { Fig. 8M } \\ \text { IB } & \text { Osprey Islet, } & \end{array}$

Bird Island inside

of Blue Lagoon

Palfrey Island,

Loomis Reef,

Horseshoe Reef,

North Point

HW Blue Lagoon,

Bird Island,

Horseshoe Reef

HW

IB

LIRS, aquarium

Loomis Reef,

Horseshoe Reef

HW

South Island

intertidal, coral

rubble in

Blue Lagoon,

Coconut Beach

intertidal,

Casuarina Beach

Coconut Beach,

Casuarina Beach

HW Coral rubble in Blue

IB Lagoon, bommies in

Blue Lagoon,

Mangrove Beach

intertidal,

Loomis Beach

Casuarina Beach,

Mangrove Beach,

Palfrey Island

Blue Lagoon,

Casuarina Beach,

Coconut Beach

Loomis,

Horseshoe Reef

$\begin{array}{ll}\begin{array}{l}\text { Polybranchia } \\ \text { orientalis }\end{array} & \text { July } 1999 \\ \text { (Kelaart, 1858) } & \text { July 2002 } \\ \text { Sept. 2002 } \\ \text { P. cf. orientalis } & \text { Aug.-Sept. 2004 }\end{array}$

HW

IB

South Island

intertidal Coral

rubble in Blue

Lagoon off

Bird Island

Coconut Beach

intertidal

Coconut Beach

$\begin{array}{lll}\text { Thuridilla } & \text { July 2002 } & \text { HW } \\ \text { carlsoni } & \text { Jan. 2004 } & \text { IB } \\ \text { Gosliner, 1995 } & \text { Aug.-Sept. 2004 } & \end{array}$

Coral rubble in

Blue Lagoon

Horseshoe Reef

Loomis Reef,

Bird Is

\section{Wägele and \\ Klussmann-Kolb, 2005}

Fig. 8N

Fig. 9A

Wägele and

Johnson 2001

Wägele and

Klussmann-Kolb, 2005

Wägele 2004

Fig. 9B

Fig. 9C 


\begin{tabular}{|c|c|c|c|c|c|c|}
\hline $\begin{array}{l}\text { Higher } \\
\text { Hierarchy }\end{array}$ & Species & $\begin{array}{l}\text { Date of } \\
\text { Observation }\end{array}$ & $\begin{array}{l}\text { Loc. of } \\
\text { material }\end{array}$ & $\begin{array}{l}\text { Site and } \\
\text { Habitat }\end{array}$ & $\begin{array}{l}\text { New to } \\
\text { GBR }\end{array}$ & $\begin{array}{l}\text { Figured and/or } \\
\text { published in: }\end{array}$ \\
\hline & $\begin{array}{l}\text { Thuridilla gracilis } \\
\text { (Risbec, 1928) }\end{array}$ & $\begin{array}{l}\text { July } 1999 \\
\text { Jan./Feb. } 2003 \\
\text { June } 2003 \\
\text { Jan. } 2003 \\
\text { Aug.-Sept. } 2004\end{array}$ & $\begin{array}{l}\text { HW } \\
\text { IB }\end{array}$ & \multicolumn{2}{|l|}{$\begin{array}{l}\text { Ghost Beach } \\
\text { intertidal, } \\
\text { Off Casuarina } \\
\text { Beach, Mermaid } \\
\text { Cove, Ghost Beach, } \\
\text { Casuarina Beach, } \\
\text { Coconut Beach, } \\
\text { Mangrove Beach } \\
\text { Blue Lagoon, } \\
\text { Horseshoe Reef } \\
\text { Loomis Reef, } \\
\text { Coconut Beach, } \\
\text { Bird Island, } \\
\text { Horseshoe Reef, } \\
\text { Washing Machine, } \\
\text { North Point }\end{array}$} & $\begin{array}{l}\text { Fig. } 9 F \\
\text { Wägele and } \\
\text { Johnson } 2001 \\
\text { as T. ratna; }\end{array}$ \\
\hline & $\begin{array}{l}\text { Thuridilla kathae } \\
\text { Gosliner } 1995\end{array}$ & Aug.-Sept. 2004 & IB & \multicolumn{2}{|l|}{ Loomis Reef } & Fig. 9D \\
\hline & $\begin{array}{l}\text { Thuridilla livida } \\
\text { (Baba, 1955) }\end{array}$ & Aug.-Sept. 2004 & IB & Bird Island & + & Fig. 9E \\
\hline & $\begin{array}{l}\text { Thuridilla } \\
\text { multimarginata } \\
\text { Gosliner, } 1995\end{array}$ & Jan. 2004 & & \multicolumn{2}{|l|}{$\begin{array}{l}\text { Blue lagoon } \\
\text { patch reef }\end{array}$} & Fig. 9H \\
\hline & $\begin{array}{l}\text { Thuridilla neona } \\
\text { Gosliner, } 1995\end{array}$ & Aug.-Sept. 2004 & IB & \multicolumn{2}{|l|}{ Bird Island } & Fig. 9G \\
\hline & $\begin{array}{l}\text { Thuridilla spec. } \\
\text { Coleman } 2001\end{array}$ & Jan. 2004 & NA & Horseshoe Reef & $?$ & Fig. 4E \\
\hline Pleurobran & $\begin{array}{l}\text { ea } \\
\text { Berthellina citrina } \\
\text { (Rüppell and } \\
\text { Leuckart, 1828) }\end{array}$ & $\begin{array}{l}\text { July } 1999 \\
\text { Aug.-Sept. } 2004\end{array}$ & $\begin{array}{l}\text { HW } \\
\text { IB }\end{array}$ & \multicolumn{2}{|l|}{$\begin{array}{l}\text { Ghost Beach } \\
\text { intertidal, } \\
\text { Coconut Beach, } \\
\text { Bird Island }\end{array}$} & $\begin{array}{l}\text { Fig. 9K } \\
\text { Wägele and } \\
\text { Klussmann-Kolb, } \\
2005\end{array}$ \\
\hline & $\begin{array}{l}\text { Berthella stellata } \\
\text { (Risso, 1826) }\end{array}$ & July 1999 & $\mathrm{HW}$ & \multicolumn{2}{|l|}{$\begin{array}{l}\text { Intertidal, coral } \\
\text { rubble in } \\
\text { Blue Lagoon }\end{array}$} & Fig. 9I \\
\hline & $\begin{array}{l}\text { Pleurobranchus } \\
\text { forskalii (Ruppell } \\
\text { and Leuckart, } \\
\text { 1828) }\end{array}$ & Jan. 2004 & & \multicolumn{2}{|l|}{$\begin{array}{l}\text { Casuarina Beach } \\
\text { coral rubble }\end{array}$} & Fig. 9M \\
\hline & $\begin{array}{l}\text { Pleurobranchus } \\
\text { grandis } \\
\text { Pease, } 1868\end{array}$ & Nov. 2003 & & \multicolumn{2}{|l|}{ Watson Bay } & Fig. 9L \\
\hline \multicolumn{7}{|c|}{$\begin{array}{l}\text { Nudibranchia } \\
\text { Doridoidea }\end{array}$} \\
\hline & $\begin{array}{l}\text { Analogium } \\
\text { striatum } \\
\text { (Eliot, 1908) }\end{array}$ & Aug. 2002 & HW & \multicolumn{2}{|l|}{ Blue Lagoon } & Fig. $9 \mathrm{~N}$ \\
\hline & $\begin{array}{l}\text { Ardeadoris } \\
\text { egretta } \\
\text { Rudman, } 1984\end{array}$ & July 2002 & $\mathrm{HW}$ & \multicolumn{2}{|l|}{ North Point } & \\
\hline & $\begin{array}{l}\text { Cadlinella } \\
\text { ornatissima } \\
\text { (Risbec, 1928) }\end{array}$ & Aug.-Sept. 2004 & IB & \multicolumn{2}{|l|}{ Loomis } & Fig. 90 \\
\hline & $\begin{array}{l}\text { Ceratosoma } \\
\text { sinulata (van } \\
\text { Hasselt, 1824) }\end{array}$ & July 2002 & $\mathrm{HW}$ & \multicolumn{2}{|l|}{$\begin{array}{l}\text { Coral rubble in } \\
\text { Blue Lagoon } \\
\text { off Bird Island }\end{array}$} & \\
\hline
\end{tabular}


Table 1 (cont.)

\begin{tabular}{|c|c|c|c|c|c|c|}
\hline $\begin{array}{l}\text { Higher } \\
\text { Hierarchy }\end{array}$ & Species & $\begin{array}{l}\text { Date of } \\
\text { Observation }\end{array}$ & $\begin{array}{l}\text { Loc. of } \\
\text { material }\end{array}$ & $\begin{array}{l}\text { Site and } \\
\text { Habitat }\end{array}$ & $\begin{array}{l}\text { New to } \\
\text { GBR }\end{array}$ & $\begin{array}{l}\text { Figured and/or } \\
\text { published in: }\end{array}$ \\
\hline & $\begin{array}{l}\text { Ceratosoma tenue } \\
\text { Abraham, } 1876\end{array}$ & $\begin{array}{l}\text { Dec. } 2003 / \\
\text { Jan. } 2004\end{array}$ & & Watsons Bay & & Fig. 9P \\
\hline & $\begin{array}{l}\text { Chromodoris } \\
\text { annae Bergh, } 1877\end{array}$ & July 2002 & $\mathrm{HW}$ & $\begin{array}{l}\text { Coconut Bay } \\
\text { intertidal, } \\
\text { Mermaid Cove, } \\
\text { North Point }\end{array}$ & & Fig. 10A \\
\hline & $\begin{array}{l}\text { Chromodoris coi } \\
\text { (Risbec, 1956) }\end{array}$ & Jan. 2003 & & Blue Lagoon & & Fig. 10B \\
\hline & $\begin{array}{l}\text { Chromodoris } \\
\text { colemani } \\
\text { Rudman, } 1982\end{array}$ & $\begin{array}{l}\text { July } 1999 \\
\text { July } 2002 \\
\text { Jan. } 2004\end{array}$ & HW & $\begin{array}{l}\text { Off Bird Island } \\
\text { outer reef, } \\
\text { Coral rubble } \\
\text { Bird Islet }\end{array}$ & & Fig. 10C \\
\hline & $\begin{array}{l}\text { Chromodoris } \\
\text { daphne } \\
\text { (Angas, 1864) }\end{array}$ & July 1999 & $\mathrm{HW}$ & $\begin{array}{l}\text { Ghost Beach } \\
\text { intertidal }\end{array}$ & & Fig. 10D \\
\hline & $\begin{array}{l}\text { Chromodoris } \\
\text { decora (Pease, } \\
1860 \text { ) }\end{array}$ & $\begin{array}{l}\text { July } 2002 \\
\text { Aug. } 2002 \\
\text { Aug.-Sept. } 2004\end{array}$ & $\begin{array}{l}\mathrm{HW} \\
\mathrm{IB}\end{array}$ & $\begin{array}{l}\text { South Island } \\
\text { intertidal, } \\
\text { Coconut Beach } \\
\text { intertidal, } \\
\text { Bird Island }\end{array}$ & & Fig. 10F \\
\hline & $\begin{array}{l}\text { Chromodoris } \\
\text { elizabethina } \\
\text { Bergh, } 1877\end{array}$ & $\begin{array}{l}\text { July } 2002 \\
\text { Jan. } 2004\end{array}$ & HW & $\begin{array}{l}\text { North Point, } \\
\text { Lizard Head } \\
\text { outer crest }\end{array}$ & & Fig. 10E \\
\hline & $\begin{array}{l}\text { Chromodoris } \\
\text { fidelis (Kelaart, } \\
1858 \text { ) }\end{array}$ & $\begin{array}{l}\text { July } 1999 \\
\text { Jan. } 2004 \\
\text { Aug.-Sept. } 2004\end{array}$ & $\begin{array}{l}\text { HW } \\
\text { IB }\end{array}$ & $\begin{array}{l}\text { Coral rubble in } \\
\text { Blue Lagoon, } \\
\text { Wickies Reef } \\
\text { Loomis Reef }\end{array}$ & & Fig. 10G \\
\hline & $\begin{array}{l}\text { Chromodoris } \\
\text { geometrica } \\
\text { Risbec, } 1928\end{array}$ & $\begin{array}{l}\text { July } 2002 \\
\text { Aug.-Sept. } 2004\end{array}$ & $\begin{array}{l}\mathrm{HW} \\
\mathrm{IB}\end{array}$ & $\begin{array}{l}\text { Cobia Hole } \\
\text { Bird Island }\end{array}$ & & Fig. $10 \mathrm{H}$ \\
\hline & $\begin{array}{l}\text { Chromodoris } \\
\text { lineolata } \\
\text { (Hasselt, 1824) }\end{array}$ & $\begin{array}{l}\text { July } 1999 \\
\text { Aug.-Sept. } 2004\end{array}$ & $\begin{array}{l}\mathrm{HW} \\
\mathrm{IB}\end{array}$ & $\begin{array}{l}\text { Ghost Beach } \\
\text { intertidal, } \\
\text { Horseshoe Reef }\end{array}$ & & Fig. 101 \\
\hline & $\begin{array}{l}\text { Chromodoris } \\
\text { lochi Rudman, } \\
1982\end{array}$ & $\begin{array}{l}\text { Sept. } 2002 \\
\text { Nov. } 2003 \text { / } \\
\text { Jan. } 2004\end{array}$ & HW & $\begin{array}{l}\text { North Direction } \\
\text { Island, Coconut } \\
\text { Beach outer crest }\end{array}$ & & Fig. 10K \\
\hline & $\begin{array}{l}\text { Chromodoris } \\
\text { rubrocornuta } \\
\text { Rudman, } 1985\end{array}$ & July 1999 & $\mathrm{HW}$ & $\begin{array}{l}\text { Coral rubble in } \\
\text { Blue Lagoon }\end{array}$ & & \\
\hline & $\begin{array}{l}\text { Chromodoris } \\
\text { verrieri (Crosse, } \\
1875 \text { ) }\end{array}$ & Dec. 2003 & & Watsons Bay & & Fig. 10L \\
\hline & $\begin{array}{l}\text { Crimora lutea } \\
\text { Baba, } 1949\end{array}$ & Jan. 2003 & & Horseshoe Reef & & Fig. 10N \\
\hline & $\begin{array}{l}\text { Dendrodoris } \\
\text { cf. elongata } \\
\text { Baba, } 1936\end{array}$ & July 1999 & & locality not noted & & Fig. 10M \\
\hline & $\begin{array}{l}\text { Dendrodoris } \\
\text { nigra (Stimpson, } \\
1855 \text { ) }\end{array}$ & $\begin{array}{l}\text { July } 1999 \\
\text { July } 2002 \\
\text { Sept. } 2002 \\
\text { Aug.-Sept. } 2004\end{array}$ & $\begin{array}{l}\text { HW } \\
\text { IB }\end{array}$ & $\begin{array}{l}\text { Ghost Beach } \\
\text { intertidal, } \\
\text { coral rubble in } \\
\text { Blue Lagoon, } \\
\text { Blue Lagoon } \\
\text { Loomis Reef }\end{array}$ & & Fig. 100 \\
\hline
\end{tabular}




\begin{tabular}{|c|c|c|c|c|c|c|}
\hline $\begin{array}{l}\text { Higher } \\
\text { Hierarchy }\end{array}$ & Species & $\begin{array}{l}\text { Date of } \\
\text { Observation }\end{array}$ & $\begin{array}{l}\text { Loc. of } \\
\text { material }\end{array}$ & $\begin{array}{l}\text { Site and } \\
\text { Habitat }\end{array}$ & $\begin{array}{l}\text { New to } \\
\text { GBR }\end{array}$ & $\begin{array}{l}\text { Figured and/or } \\
\text { published in: }\end{array}$ \\
\hline & $\begin{array}{l}\text { Dendrodoris } \\
\text { tuberculosa } \\
\text { (Quoy and } \\
\text { Gaimard, 1832) }\end{array}$ & Aug.-Sept. 2004 & IB & Bird Island & & \\
\hline & Discodoris spec. & Aug. 2002 & $\mathrm{HW}$ & $\begin{array}{l}\text { Coconut Beach } \\
\text { intertidal }\end{array}$ & $?$ & Fig. 4G \\
\hline & $\begin{array}{l}\text { Glossodoris } \\
\text { atromarginata } \\
\text { (Cuvier, 1804) }\end{array}$ & $\begin{array}{l}\text { July } 2002 \\
\text { Aug. } 2002 \\
\text { Aug.-Sept. } 2004\end{array}$ & HW & $\begin{array}{l}\text { Ghost Beach, } \\
\text { Coconut Beach }\end{array}$ & & Fig. 10P \\
\hline & $\begin{array}{l}\text { Glossodoris } \\
\text { cincta (Bergh, } \\
1888 \text { ) }\end{array}$ & $\begin{array}{l}\text { July } 2002 \\
\text { Aug.-Sept. } 2004\end{array}$ & $\begin{array}{l}\text { HW } \\
\text { IB }\end{array}$ & $\begin{array}{l}\text { Blue Lagoon off } \\
\text { South Island, } \\
\text { intertidal Off } \\
\text { Casuarina Beach }\end{array}$ & & \\
\hline & $\begin{array}{l}\text { Glossodoris } \\
\text { hikuerensis } \\
\text { (Pruvot-Fol, 1954) }\end{array}$ & July 2002 & HW & Loomis Reef & & Fig. 10R \\
\hline . & $\begin{array}{l}\text { Glossodoris } \\
\text { pallida (Rüppell } \\
\text { and Leuckart, } \\
\text { 1830) }\end{array}$ & July 1999 & HW & $\begin{array}{l}\text { Off Bird Island } \\
\text { outer reef }\end{array}$ & & Fig. $10 S$ \\
\hline & Goniodoris spec. & Aug.-Sept. 2004 & IB & Loomis Reef & $?$ & Fig. 5A \\
\hline & $\begin{array}{l}\text { Gymnodoris alba } \\
\text { (Bergh, 1877) }\end{array}$ & $\begin{array}{l}\text { July } 1999 \\
\text { Dec. } 2003 \\
\text { Aug.-Sept. } 2004\end{array}$ & $\begin{array}{l}\text { HW } \\
\text { IB }\end{array}$ & $\begin{array}{l}\text { Mermaid Cove, } \\
\text { Casuarina Beach, } \\
\text { Blue Lagoon, } \\
\text { Loomis Reef, Bird } \\
\text { Island, Horseshoe } \\
\text { Reef, North Point }\end{array}$ & & Fig. 10T \\
\hline & $\begin{array}{l}\text { Gymnodoris } \\
\text { ceylonica (Kelaart, } \\
\text { 1858) }\end{array}$ & $\begin{array}{l}\text { Dec. } 2003 \\
\text { Aug.-Sept. } 2004\end{array}$ & IB & $\begin{array}{l}\text { Casuarina Beach, } \\
\text { Off Casuarina } \\
\text { Beach }\end{array}$ & & Fig. 11A \\
\hline & $\begin{array}{l}\text { Gymnodoris } \\
\text { okinawae Baba, } \\
1936\end{array}$ & July 2002 & $\mathrm{HW}$ & $\begin{array}{l}\text { Mermaid Cove } \\
\text { North Point }\end{array}$ & & Fig. 11C \\
\hline & Gymnodoris citrina & July 2002 & HW & North Point & $?$ & Fig. 11B \\
\hline & Gymnodoris spec. 1 & July 2002 & $\mathrm{HW}$ & North Point & $?$ & Fig. 5C \\
\hline & Gymnodoris spec. 2 & $\begin{array}{l}\text { July } 1999 \\
\text { Dec. } 2003\end{array}$ & HW & $\begin{array}{l}\text { Ghost Beach } \\
\text { intertidal, } \\
\text { Horseshoe Reef }\end{array}$ & $?$ & $\begin{array}{l}\text { Fig. 5D } \\
\text { Coleman } 2001\end{array}$ \\
\hline & $\begin{array}{l}\text { Halgerda } \\
\text { aurantiomaculata }\end{array}$ & June 2003 & & Cobia Hole & & \\
\hline & $\begin{array}{l}\text { Halgerda cf. } \\
\text { batangas Carlson } \\
\text { and Hoff, } 2000\end{array}$ & Aug.-Sept. 2004 & IB & North Point & $?$ & Fig. 11D \\
\hline & $\begin{array}{l}\text { Hexabranchus } \\
\text { sanguineus } \\
\text { (Rüppell and } \\
\text { Leuckart, 1830) } \\
\text { (egg clutch) }\end{array}$ & $\begin{array}{l}\text { Aug. } 2002 \\
\text { June } 2003 \\
\text { Jan. } 2004 \\
\text { Aug.-Sept. } 2004\end{array}$ & $\begin{array}{l}\mathrm{HW} \\
\mathrm{NA} \\
\mathrm{IB}\end{array}$ & $\begin{array}{l}\text { Coconut Beach } \\
\text { intertidal, } \\
\text { Casuarina Beach, } \\
\text { Watson Bay, } \\
\text { Horseshoe Reef }\end{array}$ & & Fig. 11E \\
\hline & $\begin{array}{l}\text { Hypselodoris } \\
\text { bullocki } \\
\text { (Collingwood, } \\
\text { 1881) }\end{array}$ & $\begin{array}{l}\text { July } 2002 \\
\text { Nov. } 2003\end{array}$ & HW & $\begin{array}{l}\text { North Point, } \\
\text { Mangrove Beach }\end{array}$ & & Fig. 11F \\
\hline & $\begin{array}{l}\text { Hypselodoris } \\
\text { maculosa } \\
\text { Pease, } 1871\end{array}$ & $\begin{array}{l}\text { July } 1999 \\
\text { Aug.-Sept. } 2004\end{array}$ & $\begin{array}{l}\text { HW } \\
\text { IB }\end{array}$ & $\begin{array}{l}\text { Off Bird Island } \\
\text { outer reef, } \\
\text { Horseshoe Reef }\end{array}$ & & Fig. 11G \\
\hline
\end{tabular}


Table 1 (cont.)

\begin{tabular}{|c|c|c|c|c|c|c|}
\hline $\begin{array}{l}\text { Higher } \\
\text { Hierarchy }\end{array}$ & Species & $\begin{array}{l}\text { Date of } \\
\text { Observation }\end{array}$ & $\begin{array}{l}\text { Loc. of } \\
\text { material }\end{array}$ & $\begin{array}{l}\text { Site and } \\
\text { Habitat }\end{array}$ & $\begin{array}{l}\text { New to } \\
\text { GBR }\end{array}$ & $\begin{array}{l}\text { Figured and/or } \\
\text { published in: }\end{array}$ \\
\hline & $\begin{array}{l}\text { Hypselodoris } \\
\text { whitei (Adams } \\
\text { and Reeve, 1850) }\end{array}$ & Aug.-Sept. 2004 & \multirow[t]{2}{*}{ IB } & \multicolumn{2}{|l|}{$\begin{array}{l}\text { Coconut Beach, } \\
\text { Bird Island }\end{array}$} & Fig. $11 \mathrm{H}$ \\
\hline & $\begin{array}{l}\text { Kaloplocamus } \\
\text { acutus Baba, } 1949\end{array}$ & Jan. 2004 & & \multicolumn{2}{|l|}{$\begin{array}{l}\text { Reef inside } \\
\text { Bird islet }\end{array}$} & Fig. $11 \mathrm{M}$ \\
\hline & $\begin{array}{l}\text { Nembrotha cristata } \\
\text { Bergh, } 1877\end{array}$ & $\begin{array}{l}\text { Apr. } 1990 \\
\text { July } 1999 \\
\text { Sept. } 2002 \\
\text { June } 2003 \\
\text { Jan. } 2004 \\
\text { Aug.-Sept. } 2004\end{array}$ & $\begin{array}{l}\text { HW } \\
\text { IB }\end{array}$ & \multicolumn{2}{|l|}{$\begin{array}{l}\text { Loomis Beach, } \\
\text { Horseshoe Reef, } \\
\text { Bird Island Reef, } \\
\text { Blue Lagoon, } \\
\text { Casuarina Beach, } \\
\text { Loomis Reef, } \\
\text { Bird Island }\end{array}$} & Fig. 11I \\
\hline & $\begin{array}{l}\text { Nembrotha } \\
\text { kubaryana } \\
\text { Bergh, } 1877\end{array}$ & $\begin{array}{l}\text { July 1999, } \\
\text { July } 2002 \\
\text { Aug./Sept. } 2002 \\
\text { Jan. } 2003 \\
\text { June } 2003 \\
\text { Nov. } 2003 \\
\text { Aug.-Sept. } 2004\end{array}$ & $\begin{array}{l}\mathrm{HW} \\
\mathrm{IB}\end{array}$ & \multicolumn{2}{|l|}{$\begin{array}{l}\text { Ghost Beach, } \\
\text { Horseshoe Reef, } \\
\text { off Bird Island } \\
\text { outer reef, North } \\
\text { Point, Blue Lagoon, } \\
\text { Loomis Reef, Bird } \\
\text { Island inner reef, } \\
\text { Washing Machine, } \\
\text { Palfrey Island, } \\
\text { Coconut Beach, } \\
\text { Bird Island, } \\
\text { Horseshoe Reef }\end{array}$} & $\begin{array}{l}\text { Fig. 11K } \\
\text { Wägele and } \\
\text { Johnson } 2001\end{array}$ \\
\hline & $\begin{array}{l}\text { Nembrotha } \\
\text { lineolata } \\
\text { Bergh, } 1905\end{array}$ & Aug. 2002 & HW & \multicolumn{2}{|l|}{ Bird Island Reef } & Fig. 11L \\
\hline & $\begin{array}{l}\text { Nembrotha } \\
\text { milleri Gosliner } \\
\text { and Behrens, } 1997\end{array}$ & Nov./Dec. 2003 & & \multicolumn{2}{|l|}{ Casuarina Beach } & \\
\hline & $\begin{array}{l}\text { Notodoris citrina } \\
\text { Bergh, } 1875\end{array}$ & Aug.-Sept. 2004 & IB & \multicolumn{2}{|l|}{ Horseshoe Reef } & Fig. $11 \mathrm{~N}$ \\
\hline & $\begin{array}{l}\text { Notodoris } \\
\text { gardineri Eliot, } \\
1903\end{array}$ & $\begin{array}{l}\text { July } 1999 \\
\text { July } 2002 \\
\text { Jan. } 2003 \\
\text { June } 2003 \\
\text { Jan. } 2004\end{array}$ & HW & \multicolumn{2}{|l|}{$\begin{array}{l}\text { Osprey Islet, } \\
\text { Mermaid cove, off } \\
\text { Bird Island outer } \\
\text { reef, North Point, } \\
\text { Turtle Beach, } \\
\text { Horseshoe Reef, } \\
\text { South Island, } \\
\text { Coconut Beach, } \\
\text { Blue Lagoon }\end{array}$} & Fig. $110, \mathrm{P}$ \\
\hline & $\begin{array}{l}\text { Notodoris minor } \\
\text { Eliot, } 1904\end{array}$ & $\begin{array}{l}\text { July } 2002 \\
\text { Jan. } 2003 \\
\text { Mar. } 1990 \\
\text { June } 2003 \\
\text { Jan. } 2004 \\
\text { Aug.-Sept. } 2004\end{array}$ & $\begin{array}{l}\text { HW } \\
\text { IB }\end{array}$ & \multicolumn{2}{|l|}{$\begin{array}{l}\text { Off Bird Island } \\
\text { outer reef, } \\
\text { Mermaid Cove } \\
\text { Horseshoe Reef, } \\
\text { Blue Lagoon, } \\
\text { North Point }\end{array}$} & Fig. 11R \\
\hline & $\begin{array}{l}\text { Noumea crocea } \\
\text { Rudman, } 1986\end{array}$ & Aug.-Sept. 2004 & IB & \multicolumn{2}{|l|}{ Bird Island } & Fig. 11S \\
\hline & $\begin{array}{l}\text { Noumea romeri } \\
\text { Risbec, } 1928\end{array}$ & July 1999 & HW & \multicolumn{2}{|l|}{ Osprey Islet } & Fig. 12A \\
\hline & $\begin{array}{l}\text { Noumea varians } \\
\text { (Pease, 1871) }\end{array}$ & Aug.-Sept. 2004 & IB & \multicolumn{2}{|l|}{ Horseshoe Reef } & Fig. 12B \\
\hline & $\begin{array}{l}\text { Pectenodoris } \\
\text { trilineata (Adams } \\
\text { and Reeve, 1850) }\end{array}$ & Aug.-Sept. 2004 & IB & \multicolumn{2}{|l|}{ Bird Island } & Fig. 12C \\
\hline
\end{tabular}




\begin{tabular}{|c|c|c|c|c|c|c|}
\hline $\begin{array}{l}\text { Higher } \\
\text { Hierarchy }\end{array}$ & Species & $\begin{array}{l}\text { Date of } \\
\text { Observation }\end{array}$ & $\begin{array}{l}\text { Loc. of } \\
\text { material }\end{array}$ & $\begin{array}{l}\text { Site and } \\
\text { Habitat }\end{array}$ & $\begin{array}{l}\text { New to } \\
\text { GBR }\end{array}$ & $\begin{array}{l}\text { Figured and/or } \\
\text { published in: }\end{array}$ \\
\hline & $\begin{array}{l}\text { Phyllidia coelestis } \\
\text { Bergh, } 1905\end{array}$ & July 2002 & HW & $\begin{array}{l}\text { Bird Island outer } \\
\text { reef, Blue Lagoon }\end{array}$ & & Fig. 12E \\
\hline & $\begin{array}{l}\text { Phyllidia ocellata } \\
\text { Cuvier, } 1804\end{array}$ & $\begin{array}{l}\text { July } 2002 \\
\text { Jan. } 2004\end{array}$ & HW & $\begin{array}{l}\text { Cobia Hole, } \\
\text { Osprey islet }\end{array}$ & & Fig. 12D \\
\hline & $\begin{array}{l}\text { Phyllidia varicosa } \\
\text { Lamarck, } 1801\end{array}$ & $\begin{array}{l}\text { Oct. } 2001 \\
\text { June } 2003 \\
\text { Nov. } 2003 \\
\text { Aug.-Sept. } 2004\end{array}$ & IB & $\begin{array}{l}\text { Mac's Reef, } \\
\text { Horseshoe Reef, } \\
\text { Blue Lagoon, } \\
\text { Loomis Reef, } \\
\text { Coconut Beach }\end{array}$ & & Fig. 12F \\
\hline & $\begin{array}{l}\text { Phyllidiella lizae } \\
\text { Brunckhorst, } 1993\end{array}$ & $\begin{array}{l}\text { July } 1999 \\
\text { Aug.-Sept. } 2004\end{array}$ & $\begin{array}{l}\mathrm{HW} \\
\mathrm{IB}\end{array}$ & $\begin{array}{l}\text { Ghost Beach } \\
\text { intertidal, } \\
\text { Loomis Reef, off } \\
\text { Casuarina Beach }\end{array}$ & & Fig. $12 \mathrm{H}$ \\
\hline , & $\begin{array}{l}\text { Phyllidiella } \\
\text { pustulosa } \\
\text { (Cuvier, 1804) }\end{array}$ & $\begin{array}{l}\text { July } 1999, \\
\text { July } 2002 \\
\text { Jan./Feb. } 2003 \\
\text { June } 2003 \\
\text { Nov. } 2003 \\
\text { - Jan. } 2004 \\
\text { Aug.--Sept. } 2004\end{array}$ & $\begin{array}{l}\text { HW } \\
\text { IB }\end{array}$ & $\begin{array}{l}\text { Palfrey Island, } \\
\text { Mac's Reef, Ghost } \\
\text { Beach intertidal } \\
\text { North Point, } \\
\text { Mermaid Cove, } \\
\text { Blue Lagoon, } \\
\text { South Island } \\
\text { outer reef crest, } \\
\text { North Point }\end{array}$ & & Fig. 12G \\
\hline & $\begin{array}{l}\text { Platydoris scabra } \\
\text { (Cuvier, 1804) }\end{array}$ & $\begin{array}{l}\text { July } 1999 \\
\text { July } 2002 \\
\text { Aug./Sept. } 2002\end{array}$ & HW & $\begin{array}{l}\text { Coconut Beach } \\
\text { intertidal, }\end{array}$ & & Fig. 12I \\
\hline & $\begin{array}{l}\text { Risbecia tryoni } \\
\text { (Garrett, 1873) }\end{array}$ & $\begin{array}{l}\text { July } 1999 \\
\text { Jan. } 2003 \\
\text { June } 2003 \\
\text { Nov. } 2003 \\
\text { Aug.-Sept. } 2004\end{array}$ & $\begin{array}{l}\text { HW } \\
\text { IB }\end{array}$ & $\begin{array}{l}\text { South Island } \\
\text { intertidal } \\
\text { Blue Lagoon, } \\
\text { Palfrey Island, } \\
\text { Mangrove Beach, } \\
\text { Loomis Reef }\end{array}$ & & Fig. 12K \\
\hline & $\begin{array}{l}\text { Roboastra gracilis } \\
\text { (Bergh, 1877) } \\
\text { R. cf gracilis }\end{array}$ & $\begin{array}{l}\text { July } 1999 \\
\text { Aug. } 2002 \\
\text { Jan. } 2003 \\
\text { Nov. } 2003 \\
\text { - Jan. } 2004 \\
\text { Aug.-Sept. } 2004\end{array}$ & $\begin{array}{l}\text { HW } \\
\text { IB }\end{array}$ & $\begin{array}{l}\text { Osprey Islet, } \\
\text { Loomis Reef, } \\
\text { Off Bird Island, } \\
\text { North Point, } \\
\text { Osprey Island, } \\
\text { Palfrey Island, } \\
\text { Horseshoe Reef }\end{array}$ & & $\begin{array}{l}\text { Fig. 12L,M } \\
\text { Wägele and } \\
\text { Johnson } 2001\end{array}$ \\
\hline & $\begin{array}{l}\text { Roboastra } \\
\text { luteolineata } \\
\text { (Baba, 1936) }\end{array}$ & Dec. 2003 & & $\begin{array}{l}\text { Off South Island } \\
\text { outer Reef }\end{array}$ & & \\
\hline & $\begin{array}{l}\text { Tambja } \\
\text { limaciformis } \\
\text { (Eliot, 1908) }\end{array}$ & Oct. 2003 & & Horseshoe Reef & & Fig. 12S \\
\hline & $\begin{array}{l}\text { Tambja morosa } \\
\text { (Bergh, 1877) }\end{array}$ & July 1999 & HW & $\begin{array}{l}\text { Off Bird Island } \\
\text { outer reef }\end{array}$ & & Fig. 120 \\
\hline & Thecacera spec. & Aug. 2002 & HW & Cobia Hole & + & Fig. 5B \\
\hline & $\begin{array}{l}\text { Thorunna furtiva } \\
\text { Bergh, } 1878\end{array}$ & $\begin{array}{l}\text { July } 1999 \\
\text { July } 2002\end{array}$ & HW & $\begin{array}{l}\text { North Point } \\
\text { Blue Lagoon }\end{array}$ & & Fig. 12N \\
\hline & $\begin{array}{l}\text { Trapania cf. aurata } \\
\text { Rudman, } 1987\end{array}$ & July 2002 & HW & North Point & & Fig. 12P \\
\hline & $\begin{array}{l}\text { Trapania reticulata } \\
\text { Rudman, } 1987\end{array}$ & Aug. 2002 & HW & Bird Island Reef & & Fig. 12R \\
\hline
\end{tabular}


Table 1 (cont.)

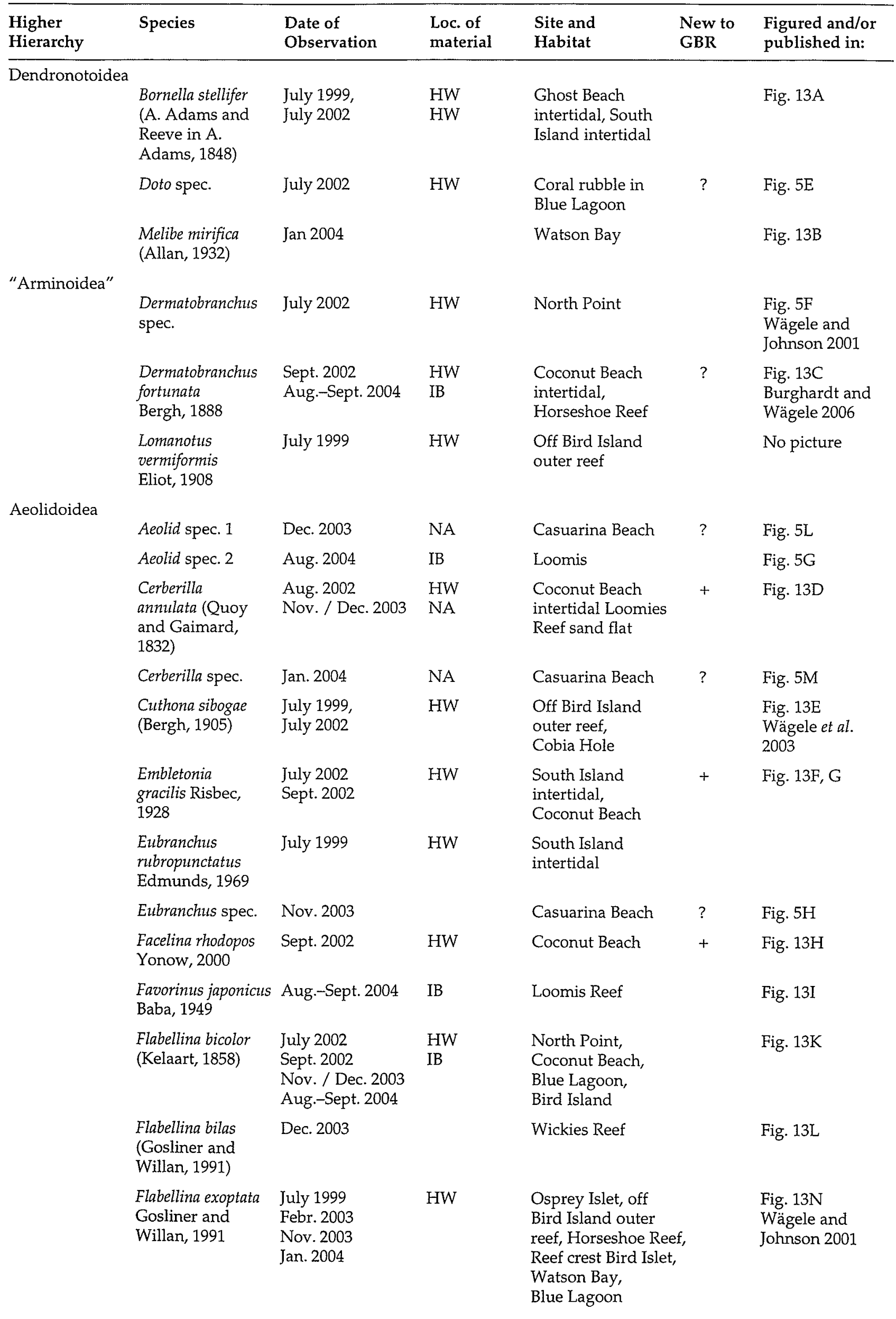




\begin{tabular}{|c|c|c|c|c|c|c|}
\hline $\begin{array}{l}\text { Higher } \\
\text { Hierarchy }\end{array}$ & Species & $\begin{array}{l}\text { Date of } \\
\text { Observation }\end{array}$ & $\begin{array}{l}\text { Loc. of } \\
\text { material }\end{array}$ & $\begin{array}{l}\text { Site and } \\
\text { Habitat }\end{array}$ & $\begin{array}{l}\text { New to } \\
\text { GBR }\end{array}$ & $\begin{array}{l}\text { Figured and/or } \\
\text { published in: }\end{array}$ \\
\hline & $\begin{array}{l}\text { Flabellina } \\
\text { rubrolineata } \\
\text { (O'Donoghue, } \\
\text { 1929) }\end{array}$ & $\begin{array}{l}\text { July } 2002 \\
\text { Jan. } 2004 \\
\text { Aug.-Sept. } 2004\end{array}$ & $\begin{array}{l}\text { HW } \\
\text { IB }\end{array}$ & \multicolumn{2}{|l|}{$\begin{array}{l}\text { North Point, } \\
\text { Coconut Beach, } \\
\text { Washing Machine }\end{array}$} & Fig. 13M \\
\hline & Godiva spec. & $\begin{array}{l}\text { Jan. } 2003 \\
\text { Oct. } 2003 \\
- \text { Jan. } 2004\end{array}$ & NA & Casuarina Beach & $?$ & $\begin{array}{l}\text { Fig. } 5 \mathrm{~K} \\
\text { Wells and } \\
\text { Bryce } 2000\end{array}$ \\
\hline & $\begin{array}{l}\text { Godiva cf. } \\
\text { quadricolor } \\
\text { (Barnard, 1927) }\end{array}$ & Jan. 2004 & & $\begin{array}{l}\text { Casuarina Beach, } \\
\text { Lizard Head }\end{array}$ & + & Fig. 13R \\
\hline & $\begin{array}{l}\text { Phestilla lugubris } \\
\text { (Bergh, 1870) }\end{array}$ & Aug.-Sept. 2004 & IB & \multicolumn{2}{|l|}{ Loomis Reef } & $\begin{array}{l}\text { Fig. } 130 \\
\text { Burghardt and } \\
\text { Wägele } 2006\end{array}$ \\
\hline & $\begin{array}{l}\text { Phidiana indica } \\
\text { (Bergh, 1896) }\end{array}$ & Jan. 2004 & & \multicolumn{2}{|l|}{ Bird Islet } & Fig. 13P \\
\hline & $\begin{array}{l}\text { Phyllodesmitum } \\
\text { briareum } \\
\text { (Bergh, 1869) }\end{array}$ & $\begin{array}{l}\text { July } 1999, \\
\text { July } 2002\end{array}$ & HW & \multicolumn{2}{|l|}{$\begin{array}{l}\text { Cobia Hole, } \\
\text { Cobia Hole, } \\
\text { North Point }\end{array}$} & $\begin{array}{l}\text { Fig. } 13 S \\
\text { Wägele and } \\
\text { Johnson } 2001 \\
\text { Wägele et al. } 2003 \\
\text { Burghardt } \text { et al. } \\
2005 \\
\text { Burghardt and } \\
\text { Wägele } 2006\end{array}$ \\
\hline & $\begin{array}{l}\text { Phyllodesmium cf. } \\
\text { hyalinum } \\
\text { Ehrenberg, } 1831\end{array}$ & Aug.-Sept. 2004 & IB & \multicolumn{2}{|l|}{$\begin{array}{l}\text { Loomis Reef, } \\
\text { Horseshoe Reef }\end{array}$} & Fig. 13U,V \\
\hline & $\begin{array}{l}\text { Phyllodesmium } \\
\text { longicirrum } \\
\text { (Bergh, 1905) }\end{array}$ & $\begin{array}{l}\text { Oct. } 2001 \\
\text { Jan. } 2003 \\
\text { June } 2003 \\
\text { Nov. } 2003 \\
\text { - Jan. } 2004\end{array}$ & NA & \multicolumn{2}{|l|}{$\begin{array}{l}\text { Washing Machine, } \\
\text { Blue Lagoon, } \\
\text { Horseshoe Reef, } \\
\text { Wickies Reef }\end{array}$} & \\
\hline & $\begin{array}{l}\text { Phyllodesmizm } \\
\text { spec. }\end{array}$ & Aug.-Sept. 2004 & IB & Bird Island & + & Fig. 51 \\
\hline & $\begin{array}{l}\text { Pteraeolidia } \\
\text { ianthina } \\
\text { (Angas, 1864) }\end{array}$ & $\begin{array}{l}\text { July } 1999 \\
\text { July } 2002 \\
\text { Sept. } 2002 \\
\text { June } 2003 \\
\text { Nov. } 2003 \\
\text { - Jan. } 2004\end{array}$ & HW & \multicolumn{2}{|l|}{$\begin{array}{l}\text { South Island, } \\
\text { Osprey Islet, } \\
\text { Coconut Beach } \\
\text { intertidal, } \\
\text { Blue Lagoon, } \\
\text { Bird Island, } \\
\text { Mangrove Beach, } \\
\text { Palfrey Island, } \\
\text { Bird Islet outer crest, } \\
\text { crest, Watson Bay }\end{array}$} & $\begin{array}{l}\text { Fig. } 13 \mathrm{~T} \\
\text { Wägele and } \\
\text { Johnson } 2001 \\
\text { Burghardt et al. } \\
2005\end{array}$ \\
\hline
\end{tabular}

Table 2 Comparison of the total number of opisthobranch species (data taken from literature) from different IndoPacific areas.

\begin{tabular}{llcl}
\hline Area & Authors & Total number of species & Collection Time Period \\
\hline Papua New Guinea & Gosliner 1992 & 538 & Not indicated \\
Great Barrier Reef & Marshall and Willan 1999 & 414 & 18 years episodic \\
Heron Island & Marshall and Willan 1999 & 261 & 18 years episodic \\
Fiji Islands & Brodie and Brodie 1990 & 251 & 4 years continuous \\
Marshall Islands & Johnson and Boucher 1983 & 101 & 3 years continuous \\
Lizard Island & Present paper & 158 & 5 years episodic \\
\hline
\end{tabular}


side of the Island were relatively under sampled because of rough weather conditions during many visits. Therefore, the species numbers presented here are preliminary in terms of the coverage of different habitat types.

Data of opisthobranch species richness from other tropical Pacific areas are available i.e. New Caledonia (Bouchet et al. 2002), Papua New Guinea (Gosliner 1992), Fiji (Brodie and Brodie 1990, 1995) and Marshall Islands (Johnson and Boucher 1983) (see Figure 2). Direct comparison is not possible, since these areas differ geographically and in size, and collection times were much longer in some studies than others (see table 2). E.g., collection on Heron Island (Great Barrier Reef) occurred over a period of 18 years with 27 field trips (Marshall and Willan 1999). Nevertheless, similar trends in the different opisthobranch subgroups are obvious for all investigated regions. All major groups of the Opisthobranchia are represented in comparable proportions and the Doridoidea are the species richest group.

Since Mikkelsen (1996, 2002) published her detailed phylogenetic analyses on the Cephalaspidea, the Acteonoidea are excluded from the Cephalaspidea, or even from the Opisthobranchia. For this reason Marshall and Willan (1999) did not include this taxon in their extensive studies on Heron Island. Therefore, in Figure 2, a zero is indicated in these two sections of their data (Great Barrier Reef column nr. 3, Heron Island column nr. 4), indicating lack of data, but not absence of this group. Gosliner (1992) did not list the Acteonoidea separately for Papua New Guinea, but the species numbers are added to the Cephalaspidea (71 species in total). All other checklists listed the Acteonoidea as a separate group as we do in this study.

Anaspidea are well represented on Lizard Island (33\% of all known Indo Pacific species - see Coleman 2001), but locality sites with high abundance of macroalgae have hardly been investigated, especially the Halimeda habitats in front of Bird Island.

58 sacoglossan species are recorded by Coleman (2001) from the Indo Pacific and Gosliner's (1992) numbers from Papua New Guinea are even higher. Twenty-one species are now recorded from Lizard Island with probably four undescribed species (Figure 4).

Tylodinoidea are only known from very few species (about 15 worldwide), and only two species (Tylodina corticalis, Umbraculum umbraculum) are recorded from Australian waters (Willan 1998). None of them were found on Lizard Island.

The highest species numbers in the Indo-Pacific are given for the Doridoidea (Coleman 2001: 420 species) and this taxon also shows the highest diversity on Lizard Island with 66 species, representing $16 \%$ of recorded Indo-Pacific dorids. Nevertheless, compared to other areas, this number is low. A thorough investigation of the sponge fauna might reveal many more species, especially cryptic ones.

The number of dendronotoidean (3) and arminoidean (3) species is low, whereas aeolidoideans are represented with more species (23). This is in line with the studies on other sites. Some Dermatobranchus species (Arminidae) are known to house zooxanthellae (Wägele and Johnsen 2001), and were specially looked for during one project on "solarpowered" seaslugs. The new Dermatobranchus species found in low depths down to $5 \mathrm{~m}$ was very small (less then $10 \mathrm{~mm}$ ) and highly cryptical due to the stored zooxanthellae.

This study is the base for further investigation on the opisthobranch biodiversity on a site, which lies between well studied areas in the South of the Great Barrier Reef (Heron Island) and the tropical Islands north of Australia (e.g. Papua New Guinea).

\section{ACKNOWLEDGEMENTS}

We would like to thank following diving and collecting partners during the different field trips: Wolfgang, Leonard and Richard Wägele (Bochum, Germany), Jon and Nicola Brodie (Townsville, Australia), Shireen Fahey (Brisbane, Australia), Nico K. Michiels (Tübingen, Germany), Geir Johnsen (Trondheim, Norway), Annika Putz (Tübingen, Germany). We also thank Alex Cheroske (Baltimore, USA), Short Chiou (Baltimore, USA), Michael Berumen (Townsville, Australia) and Onno Gross (Hamburg, Germany) for providing us with material.

We wish to express our sincere thanks to the directors of Lizard Island Research Station, Anne Hoggett and Lyle Vail, for their tremendous help and support and to their staff Tania and Bob Lamb, as well as Marianne and Lance Pearce. Richard Willan (Darwin) kindly helped in identification of several species.

This study was supported by the German Science Foundation (DFG) to Heike Wägele and Ingo Burghardt (Wa 618/5, Wa 618/6 and Wa 618/8: SPP 1127) and Nils Anthes (Mi 482/7-2). Further support came from the Deutscher Akademischer Austauschdienst (DAAD) to Nils Anthes (D/02/28302), Freunde und Förderer der Ruhr-Universität Bochum to Annette Klussmann$\mathrm{Kolb}$, the Department of Marine Biology (JCU) to Gilianne Brodie and the Australian Biological Resources Study (ABRS) to Gilianne Brodie and Heike Wägele. Jussi Evertson was supported by the Norwegian Science Foundation (NFR 153790/ 120). The manuscript is contribution number 25 of the Marine Invertebrate Group at James Cook University. 


\section{REFERENCES}

Anthes, N., Putz, A. and Michiels, N. K. (2005). Gender trading in a hermaphrodite. Current Biology 15: R792R793.

Anthes, N., Putz, A. and Michiels, N. K. (2006). Hermaphrodite sex role preferences: the role of partner body size, mating history and female fitness in the sea slug Chelidonura sandrana. Behavioural Ecology and Sociobiology 60, in press (doi 10.1007/ s00265-006-0173-5).

Anthes, N. and Michiels, N. K. (2005). Do "sperm trading" simultaneous hermaphrodites always trade sperm? Behavioral Ecology 16: 188-195

Bouchet, P., Lozouet, P., Maestrati, P. and Heros, V. (2002). Assessing the magnitude of species richness in tropical marine environments: exceptionally high numbers of molluscs at a New Caledonia site. Biological Journal of the Linnean Society 75: 421-436.

Brodie, G.D. and Brodie, J.E. (1990). A checklist of the opisthobranch molluscs of Fiji. Journal of the Malacological Society of Australia 11: 53-63.

Brodie, G.D. and Brodie, J.E. (1995). Species diversity and habitat selection in opisthobranch gastropods on two adjacent reefs in Fiji. South Pacific Journal of Natural Science 14: 97-113.

Burghardt, I. and Wägele, H. (2006). Interspecific differences in the efficiency and photosynthetic characteristics of the mutualistic symbiosis of "solarpowered" Nudibranchia (Mollusca: Gastropoda) with zooxanthellae. Records of the Western Australian Museum Supplement 69: 1-9.

Burghardt, I., Evertsen, J., Johnsen, G. and Wägele, H. (2005). Mutualistic symbiosis of aeolid Nudibranchia (Mollusca, Gastropoda, Opisthobranchia) with zooxanthellae of the genus Symbiodinium. Symbiosis 38: 227-250.

Chadwick, V. and Green, A. (2002). Managing the Great Barrier Reef Marine Park and World Heritage Area through Critical Issues Management: Science and Management. In: Moosa et al. (eds.) Proceedings of the $g^{\text {th }}$ International Coral Reef Symposium. October, 2000, Bali, Indonesia. 2: 681-686.

Coleman, N. (2001): 1001 Nudibranchs. Catalogue of IndoPacific Sea slugs. Neville Coleman's Underwater Geographic Pty Ltd. 144 pp.

Done, T.J., Ogden, J.C., Wiebe, W.J. and Rosen, B.R. (1996). Biodiversity and ecosystem function of coral reefs. pp. 393-429. In: Heywood VH (ed) Global biodiversity assessment. Cambridge University Press for United Nations Environment Programme, 1152 p.

Gosliner, T.M. (1992). Biodiversity of tropical opisthobranch gastropod faunas. Proceedings of the $7^{\text {th }}$ International Coral Reef Symposium, Guam, 2: 702-709.
Gosliner, T.M. and Draheim, R. (1996). Indo-Pacific opisthobranch gastropod biogeography: how do we know what we don't know? American Malacological Bulletin 12: 37-43.

Johnson, S. and Boucher, L.M. (1983). Notes on some Opisthobranchia (Mollusca: Gastropoda) from the Marshall Islands including 57 new records. Pacific Science 37: 251-291.

Marshall, J.G. and Willan, R.C. (1999). Nudibranchs of Heron Island, Great Barrier Reef. Backhuys Publishers Leiden. $257 \mathrm{pp}$

Mikkelsen, P.M. (1996). The evolutionary relationships of Cephalaspidea s.l. (Gastropoda: Opisthobranchia): a phylogenetic analysis. Malacologia 37: 375-442.

Mikkelsen, P.M. (2002). Shelled opisthobranchs. Advances in Marine Biology 42: 67-136.

Mikkelsen, P.M. and Cracraft, J. (2001). Marine biodiversity and the need for systematic inventories. Bulletin of Marine Science 69: 525-534.

Vonnemann, V., Schrödl, M. Klussmann-Kolb, A. and Wägele, H. (2005). Reconstruction of the phylogeny of the Opisthobranchia (Mollusca, Gastropoda) by means of $18 \mathrm{~S}$ and $28 \mathrm{~S}$ rRNA gene sequences. Journal of Molluscan Studies 71: 113-125.

Wägele, H., Vonnemann, V. and Wägele J.W. (2003). Toward a phylogeny of the Opisthobranchia. 185-228 In: Lydeard, C. and Lindberg, D. (eds.) Molecular systematics and phylogeography of mollusks. Smithsonian Institution Press. 312 pp.

Wägele, H. and Johnsen, G. (2001). Observations on the histology and photosynthetic performance of "solarpowered" opisthobranchs (Mollusca, Gastropoda, Opisthobranchia) containing symbiotic chloroplasts or zooxanthellae. Organisms, Diversity and Evolution 1: 193-210

Wägele, H. (2004). Potential key characters in Opisthobranchia (Gastropoda, Mollusca) enhancing adaptive radiation. Organisms, Diversity and Evolution 4: 175-188.

Wägele, H. and Klussmann-Kolb, A. (2005). Opisthobranchia (Mollusca, Gastropoda) - more than just slimy slugs. Shell reduction and its implications on defence and foraging. Frontiers in Zoology 2: 3.

Wells, F.E. and C.W. Bryce (2000). Sea shugs of Western Australia. A guide to species from the Indian to the Wes Pacific Oceans. Western Australian Museum, Perth. 184 pp.

Willan, R.C. and Coleman, N. (1984). Nudibranchs of Australasia. National Library of Australia. $56 \mathrm{pp}$.

Willan, R.C. (1998). Order Notaspidea. Pp 977-980 in Beesley, P.L., Ross, G.j.B. and Wells, A. (eds) Mollusca: The Southern Synthesis. Fauna of Australia. Vol. 5. CSIRO Publishing: Melbourne, Part B viii 565-1234 pp. 